\title{
Utilidad del carbonato de litio, yoduro inorgánico y plasmaféresis en una paciente con hipertiroidismo severo y agranulocitosis por tiamazol en tiempos de COVID-19 \\ Usefulness of lithium carbonate, inorganic iodide and plasmapheresis in a patient with severe hyperthyroidism and agranulocytosis due to thiamazole in times of COVID-19
} \author{
José Luis Paz-Ibarra ${ }^{1,2, e}$ \\ ${ }^{1}$ Servicio de Endocrinologia, Hospital Nacional Edgardo Rebagliati Martins. Lima, Perú. \\ ${ }^{2}$ Facultad de Medicina, Universidad Nacional Mayor de San Marcos. Lima, Perú. \\ ${ }^{a}$ Médico residente de endocrinología, ORCID: https://orcid.org/0000-0002-6846-7630 \\ ${ }^{b}$ Médico residente de endocrinologia, ORCID: https://orcid.org/0000-0002-6861-5699 \\ 'Médico residente de endocrinologia, ORCID: https://orcid. org/0000-0002-2064-6509 \\ ${ }^{\mathrm{d}}$ Médico endocrinóloga, ORCID: https://orcid.org/0000-0001-7779-1654 \\ ${ }^{e}$ Médico endocrinólogo, ORCID: https://orcid.org/0000-0002-2851-3727
}

Víctor Raúl García-Ruiz ${ }^{1, a}$, Julio César Álvarez-Gamero ${ }^{1, b}$, Fernando Quinto-Reyes ${ }^{1, c}$, María Pilar Churampi-López ${ }^{1,2, d}$,

An Fac med. 2020;81(3):333-6. / DOI: https://doi.org/10.15381/anales.v81i3.19442

Correspondencia:
Victor Raúl Garcia Ruiz
vrsgarcia16@gmail.com

Recibido: 23 de octubre 2020

Aprobado: 13 de diciembre 2020

Publicación en línea: 30 de diciembre 2020

Conflictos de interés: Los autores declaran no tener conflictos de interés.

Fuente de financiamiento:

Autofinanciado

Contribuciones de autoría: Todos los autores participaron en la recolección de resultados; análisis e interpretación de datos; redacción del artículo; revisión critica del artículo; aprobación de la versión final y aporte material de estudio.

Citar como: García-Ruiz V, AlvarezGamero J, Quinto-Reyes F, ChurampiLópez M, Paz-Ibarra J. Utilidad del carbonato de litio, yodo inorgánico y plasmaféresis en una paciente con hipertiroidismo severo y agranulocitosis por tiamazol en tiempos de COVID-19. An Fac med. 2020;81(3):333-6.

DOI: https://doi.org/10.15381/anales.

v81i3.19442

\section{Resumen}

Mujer de 43 años con diagnóstico reciente de enfermedad de Graves-Basedow ingresa a urgencias por infección de tejidos blandos, agranulocitosis por tiamazol e hipertiroidismo descompensado. La paciente requeria tratamiento definitivo para hipertiroidismo, pero dado el contexto de la pandemia por SARS-CoV-2 no se contaba con yodo radioactivo ${ }^{(131}$ I) por lo que se planteó la tiroidectomía total. Se necesitaba una preparación preoperatoria rápida y eficaz por lo que se decidió compensar su hipertiroidismo con lugol, carbonato de litio, dexametasona y finalmente plasmaféresis antes de cirugía. Con ello se logró disminuir los niveles de hormonas tiroideas en el tiempo deseado y se procedió al tratamiento quirúrgico definitivo. Se presenta el caso por lo anecdótico y por ser necesario el conocimiento del manejo en escenarios, como esta pandemia, donde no haya acceso a ${ }^{131} \mathrm{l}$.

Palabras clave: Agranulocitosis; Metimazol; Hipertiroidismo; COVID-19 (fuente: DeCS BIREME).

\section{Abstract}

A 43-year-old woman with a recent diagnosis of Graves-Basedow disease was admitted to the emergency room due to soft tissue infection, thiamazole agranulocytosis, and severe hyperthyroidism. The patient required definitive treatment for hyperthyroidism, but given the context of the SARS-COV-2 pandemic, radioactive iodine was not available, so she prepared for total thyroidectomy. A rapid and effective preoperative preparation was required, so it was decided to compensate her hyperthyroidism with lugol, lithium carbonate, dexamethasone and finally plasmapheresis before surgery. Thyroid hormone levels normalized in a short time, and the thyroidectomy was performed. The case is presented because of the anecdotal and because knowledge of management in scenarios, such as this pandemic, where there is no access to ${ }^{131}$ I.

Keywords: Agranulocytosis; Methimazole; Hyperthyroidism; COVID-19 (source: MeSH NLM). 


\section{INTRODUCCION}

La pandemia por el nuevo virus SARSCoV- 2 constituye un reto para los sistemas de salud, significando que muchos de los recursos sean destinados a su prevención y tratamiento comprometiendo de manera indirecta la atención de otras afecciones agudas y crónicas en los diferentes centros de atención. El hipertiroidismo es una enfermedad que se caracteriza por la síntesis excesiva de hormonas tiroideas por la glándula tiroides con una prevalencia estimada entre 0,2 a $1,3 \%$ en áreas de yodo suficiencia ${ }^{(1)}$ y de $1,2 \%$ según reportes de USA ${ }^{(2)}$, siendo la enfermedad de Graves-Basedow (EGB) la causa más frecuente ${ }^{(3)}$. La terapia médica con antitiroideos de síntesis, ya sea con tiamazol, o propiltiuracilo, constituye una de las primeras opciones terapéuticas ${ }^{(2)}$. La agranulocitosis por tiamazol se presenta en el $0,1 \%$ a $0,3 \%{ }^{(2)}$ de los casos y supone una situación de emergencia que requiere de manejo especializado, así como también representa una contraindicación absoluta para el empleo de antitiroideos de síntesis por lo que debe optarse por alternativas terapéuticas tales como radioyodo o tiroidectomía. Presentamos el caso de una paciente con sepsis de foco dérmico y respiratorio, hipertiroidismo no controlado y agranulocitosis inducida por tionamidas, la cual ingresó al hospital durante la emergencia sanitaria, y no pudo recibir tratamiento con radioyodo por falta de su producción en el país, por estado de emergencia sanitaria, por lo que se decidió su preparación para tiroidectomía. Se presenta el caso por lo anecdótico y por las circunstancias que pueden ocurrir a nivel mundial por esta pandemia u otras que dificulten el acceso a yodo radioactivo.

\section{REPORTE DE CASO}

Mujer de 43 años ingresó a la emergencia a finales de mayo del 2020 por cuadro febril asociado a un aumento de volumen en hemicara derecha, dolor de garganta y dificultad para respirar. Refirió diagnóstico reciente de hipertiroidismo por enfermedad de Graves-Basedow en tratamiento con tiamazol $40 \mathrm{mg} /$ día, que estuvo tomando tres semanas previas a su ingreso. Al examen físico: temperatura $38,8^{\circ} \mathrm{C}$, frecuencia cardiaca 118 por minuto, frecuencia respiratoria 26 por minuto con saturación de oxigeno $92 \%$, presión arterial: 120/70 $\mathrm{mmHg}$, peso 38 kg, IMC 14,8, piel caliente y húmeda, con signos de flogosis a nivel maxilar derecho, bocio grado 2, crepitantes en base de pulmón derecho y tremor distal marcado. Resto de examen sin alteraciones. Se evidencia escala de Burch y Wartofsky: 20 (baja probabilidad de tormenta tiroidea) y un electrocardiograma con taquicardia sinusal.

La analítica al ingreso se resume en la tabla 1, donde se destacó: leucocitos: $890 \times \mathrm{mm}^{3}$ (VN 4000-11000), neutrófilos $71 \times \mathrm{mm}^{3}$ (VN 1800-8100), hemoglobina 8,2 g/dL (VN 12-16), perfil hepático: albumina 2,6 g/dL (VN 3,2-4,8), TGO $27 \mathrm{U} / \mathrm{L}$ (VN 0-34), TGP $18 \mathrm{U} / \mathrm{L}$ (VN 10-49), bilirrubina totales 1,1 mg/dL (VN 0,3-1,2), PCR 16,6 mg/dL (VN 0-1), TSH 0,015 uUI/L (VN 0,4-4), T4L 4,66 ng/dL (VN 0,8-1,9), T3L 8,9 pg/mL (VN 1,8-4,2), anti TPO: $246 \mathrm{IU} / \mathrm{mL}$. Tomografía sin contraste que informó signos inflamatorios de hemicara derecha hacia arcada superior lateral, sinusitis maxilar a predominio derecho y consolidado pulmonar basal derecho. UItrasonografía tiroidea: bocio difuso con doppler compatible con "infierno tiroideo". La presencia de autoinmunidad tiroidea y las características de la ecografía tiroidea confirmaron el antecedente de enfermedad de Graves-Basedow.

Se hospitalizó con diagnóstico de celulitis en hemicara derecha, neumonía adquirida en la comunidad, agranulocitosis por tiamazol. Se suspendió antitiroideos, se administró cobertura antibiótica amplia por 14 días (meropenem y vancomicina), propranolol $40 \mathrm{mg}$ cada 6 horas y factor estimulante de colonias de granulocitos 30000 ME por 5 dosis, con evolución favorable del proceso infeccioso y remisión de la neutropenia. Sin embargo, persistió con sintomatología propia de hipertiroidismo: taquicardia (100-114 por minuto), temperatura $37,7^{\circ}$, debilidad proximal y tremor distal. Debido a que no se contaba con disponibilidad de radioyodo ( ${ }^{131} 1$ ), por no existir producción local por el Instituto Peruano de Energía Nuclear debido a la emergencia sanitaria nacional por la pandemia COVID-19, se planteó manejo quirúrgico como tratamiento definitivo del hipertiroidismo. Se realizó preparación pre-quirúrgica con lugol al 5\% 5 gotas c/8 horas, dexametasona $1 \mathrm{mg} \mathrm{c} / 18$ horas VO y carbonato de litio 300mg c/8 horas VO, objetivándose disminución de los niveles de hormonas tiroideas después de 7 días (figuras 1 y 2). Finalmente, en vista de limitación de turno operatorio por el estado de emergencia, se decidió que sea sometida a dos sesiones de plasmaféresis en días consecutivos, lográndose normalización de hormonas tiroideas, siendo sometida a tiroidectomía total que toleró sin complicaciones; siendo dada de alta al quinto día postoperatorio con levotiroxina 50 ug/día por una semana y luego 75 ug/día asociado a suplementos de calcio y calcitriol y valores de hormonas tiroideas T4L $0,45 \mathrm{ng} / \mathrm{dL}, \mathrm{T} 3 \mathrm{~L} 1,6 \mathrm{pg} / \mathrm{mL}$ (figuras 1 y 2 ).

\section{DISCUSIÓN}

La enfermedad de Graves-Basedow es la primera causa de hipertiroidismo primario en el mundo. El manejo médico con tionamidas (tiamazol, propiltiuracilo) suele ser de elección en pacientes jóvenes, mujeres, con enfermedad leve,

Tabla 1. Exámenes de laboratorio según día de hospitalización en paciente con hipertiroidismo severo y agranulocitosis por tiamazol.

\begin{tabular}{lcccc} 
Hemograma & Ingreso & Día 1 & Día 2 & Día 3 \\
\hline Leucocitos (VN 4000-11000/uL) & 8750 & 890 & 1540 & 4610 \\
\hline Neutrófilos (VN 1800-8000/uL) & 262 & 71 & 300 & 1230 \\
\hline Hemoglobina (VN 14-18 g/dL) & 11,1 & 8,2 & 9,5 & 8,5 \\
\hline Plaquetas (VN 130 000- 400 000/uL) & 415000 & & 215000 & 215000 \\
\hline Potasio (VN 3,5-5,5 mmol/L) & & 3,24 & 2,2 & 3,28 \\
\hline
\end{tabular}




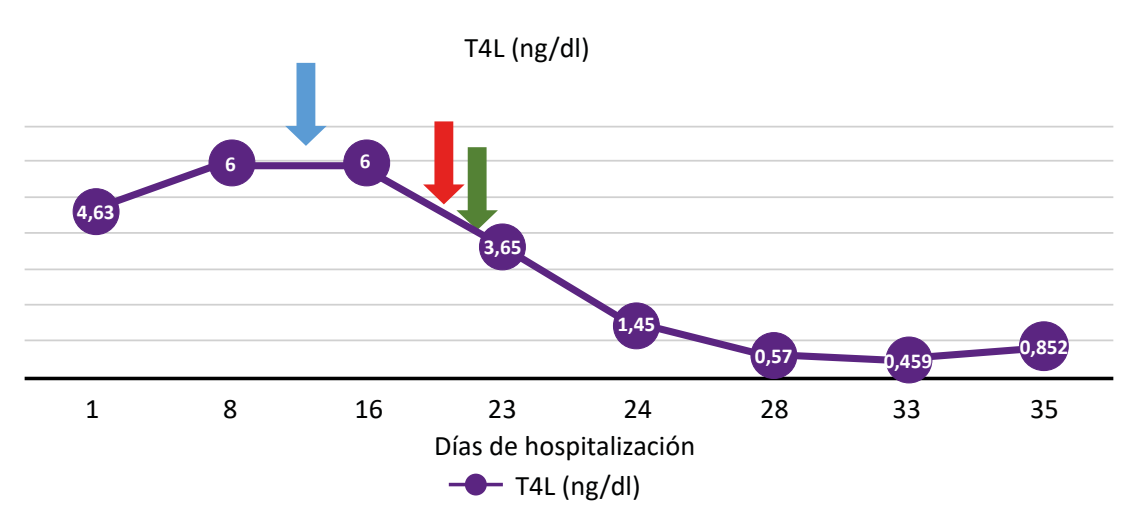

Figura 1. Variación en niveles de T4L (VN 0,8-1.9ng/dL) en paciente con hipertiroidismo severo y agranulocitosis por tiamazol.

Nótese que inició tratamiento con carbonato de litio a partir del día 12 de hospitalización (flecha azul), lugol al 5\% del día 20 (flecha roja) y plasmaféresis desde el día 22 (flecha Verde). Persistió normalización de T4L después de cirugía (día 24 en adelante).

bocio pequeño y títulos bajos de anticuerpos contra receptor de TSH (anti TRAb) ${ }^{(2)}$. La agranulocitosis presentada en la paciente es un efecto adverso infrecuente pero grave del tiamazol, que obliga a su suspensión definitiva ${ }^{(4)}$. El tratamiento alternativo con ${ }^{131}$ I es eficaz en este contexto de reacciones adversas severas por tiamazol; sin embargo, no estaba disponible por falta de su producción por estado de emergencia en nuestro país por SARS-COV-2.

El tratamiento quirúrgico es otra opción cuando no es posible el manejo con tionamidas y radioyodo ${ }^{(2)}$. La preparación pre-quirúrgica en estos pacientes tiene como finalidad alcanzar un estado eutiroideo para evitar complicaciones durante la cirugía ${ }^{(2)}$. En cirugías electivas la preparación suele ser con tionamidas y la duración promedio de tratamiento en espera de cirugía es hasta de 6 meses según la serie de casos publicada por Daruoassi (5); pero cabe resaltar que según otra revisión, la duración promedio para lograr el eutiroidismo es 3 a 12 semanas ${ }^{(6)}$. Cuando no se puede usar tiamazol, principalmente por presentar efectos adversos severos, se han descrito esquemas alternativos con

\section{$\mathrm{T} 3(\mathrm{pg} / \mathrm{ml})$}

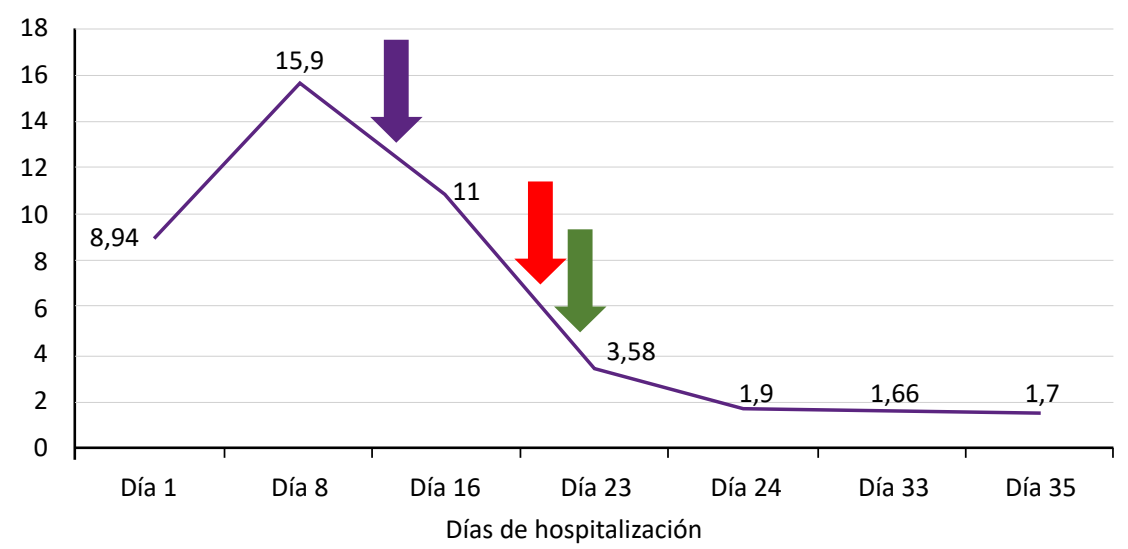

Figura 2. Variación en niveles de T3L (VN 1,8-4,2 pg/mL) en paciente con hipertiroidismo severo y agranulocitosis por tiamazol.

Nótese que inició tratamiento con carbonato de litio a partir del día 12 de hospitalización (flecha azul), lugol al 5\% del día 20 (flecha roja) y plasmaféresis desde el día 22 (flecha Verde). Persistió normalización de T3L después de cirugía (día 24 en adelante). carbonato de litio, dexametasona y betabloqueadores ${ }^{(7)}$.

Se sabe que el litio se concentra 3 a 4 veces más en la tiroides que en el plasma. Al administrase en humanos, reduce la captación tiroidea de iodo e interrumpe la liberación de las hormonas tiroideas tanto en eutiroideos como hipertiroideos ${ }^{(8)}$. Asimismo, se ha descrito que el tratamiento con carbonato de litio a dosis de $300 \mathrm{mg}$ cada 8 horas son efectivas para el control de tirotoxicosis cuando no se puede prescribir tionamidas ${ }^{(9)}$. El tratamiento combinado con lugol se realizó por su efecto sobre la vascularización de la glándula, menor riesgo de sangrado intraoperatorio ${ }^{(10)}$, su capacidad para bloquear la organificación del iodo y disminuir así la síntesis de hormonas tiroidea para lograr el eutiroidismo ${ }^{(11)}$.

La pandemia por SARS-CoV-2 provocó que el sistema de salud enfocara todos los recursos al manejo de la infección, limitando la disponibilidad de salas quirúrgicas y ventiladores mecánicos, por lo que el procedimiento quirúrgico de la paciente debía hacerse a la brevedad. Este caso representó un reto en el manejo de la paciente por la necesidad de resolver la tirotoxicosis en el menor tiempo posible. Si bien el tratamiento combinado de carbonato de litio, lugol y dexametasona mostraba un descenso de las hormonas tiroideas, se planteó el uso de plasmaféresis ante la necesidad de operar a la brevedad a la paciente. Si bien la plasmaféresis no está recomendada como preparación prequirúrgica según las guías clínicas ${ }^{(2,12)}$, existe evidencia de su uso como una alternativa terapéutica efectiva y rápida para lograr el eutiroidismo en pacientes hipertiroideos no controlados que van a ser sometidos a tiroidectomía ${ }^{(13)}$. Este procedimiento, intercambia un volumen importante de plasma que contiene toxinas y numerosas proteínas como anticuerpos y complejos inmunes. Este intercambio también elimina hormonas tiroideas unidas a proteínas y así su reducción en plasma. En nuestro paciente sólo dos sesiones consecutivas de plasmaféresis bastaron para normalizar los niveles de hormonas tiroideas, contrario a los reportado en la literatura que suele necesitar en promedio cinco sesiones ${ }^{(14)}$; esto debido a que previamente estaba recibiendo tratamiento con litio y dexametasona; los cuales ya habían redu- 
cido el nivel de hormonas tiroideas. Todo el manejo en conjunto permitió que la paciente logre alcanzar turno operatorio y se realice la tiroidectomía, con normalización de hormonas después de la cirugia.

\section{REFERENCIAS BIBLIOGRÁFICAS}

1. Taylor PN, Albrecht D, Scholz A, Gutierrez-Buey G, Lazarus JH, Dayan CM, et al. Global epidemiology of hyperthyroidism and hypothyroidism. Nat Rev Endocrinol. 2018;14(5):301-16. DOI: 10.1038/ nrendo.2018.18

2. Ross DS, Burch HB, Cooper DS, Greenlee MC Laurberg P, Maia AL, et al. 2016 American Thyroid Association Guidelines for Diagnosis and Management of Hyperthyroidism and Other Causes of Thyrotoxicosis. Thyroid. 2016;26(10):1343-421. DOl: 10.1089/thy.2016.0229

3. Kahaly GJ, Bartalena L, Hegedüs L, Leenhardt L, Poppe K, Pearce SH. 2018 European Thyroid As- sociation Guideline for the Management of Graves' Hyperthyroidism. Eur Thyroid J. 2018;7(4):167-86. DOI: 10.1159/000490384

4. Cooper DS. Antithyroid drugs. N Engl J Med. 2005;352(9):905-917. DOI: 10.1056/NEJMra042972

5. Darouassi $Y$, Hanine MA, Aljalil A, Ennouali A, Bouaity B, Touati MM, et al. Surgical management of hyperthyroidism: about 60 cases. Pan Afr Med J. 2018;31:43. DOI:10.11604/pamj.2018.31.43.16695

6. Chiha M, Samarasinghe S, Kabaker AS. Thyroid storm: an updated review. J Intensive Care Med. 2015;30(3):131-140. DOI: $10.1177 / 0885066613498053$

7. Nair GC, C. Babu MJ, Menon R, Jacob P. Preoperative Preparation of Hyperthyroidism for Thyroidectomy - Role of Supersaturated lodine and Lithium Carbonate. Indian J Endocrinol Metab. 2018;22(3):392-6. DOI: 10.4103/ijem.IJEM_3_18

8. Lazarus JH. Lithium and thyroid. Best Pract Res Clin Endocrinol Metab. 2009;23(6):723-733. DOI: 10.1016/j.beem.2009.06.002

9. Prakash I, Nylen ES, Sen S. Lithium as an Alternative Option in Graves Thyrotoxicosis. Case Rep Endocrinol. 2015:869343. DOI: 10.1155/2015/869343
10. Tsai CH, Yang PS, Lee JJ, Liu TP, Kuo CY, Cheng SP. Effects of Preoperative lodine Administration on Thyroidectomy for Hyperthyroidism: A Systematic Review and Meta-analysis. Otolaryngo Head Neck Surg. 2019;160(6):993-1002. DOI: 10.1177/0194599819829052

11. Leung AM, Braverman LE. Consequences of excess iodine. Nat Rev Endocrinol. 2014;10(3):136-142. DOI: $10.1038 /$ nrendo.2013.251

12. Padmanabhan A, Connelly-Smith L, Aqui N, Balogun R, Klingel R, Meyer E, et al. Guidelines on the Use of Therapeutic Apheresis in Clinical Practice - Evidence-Based Approach from the Writing Committee of the American Society for Apheresis: The Eighth Special Issue. J Clin Apher. 2019;34(3):171-354. DOI: 10.1002/jca.21705

13. Ezer A, Caliskan K, Parlakgumus A, Belli S, Kozanoglu I, Yildirim S. Preoperative therapeutic plasma exchange in patients with thyrotoxicosis. J Clin Apher. 2009;24(3):111-4. DOI: 10.1002/jca.20200

14. Ozdemir Baser O, Cetin Z, Catak M, Koseoglu D, Berker D. The role of therapeutic plasmapheresis in patients with hyperthyroidism. Transfus Apher Sci. 2020;102744. DOI: 10.1016/j.transci.2020.102744 